TAO, Vol. 12, No. 3, 559-565, September 2001

\title{
The Evolution of Equatorial Trough of Ionospheric F-region Ionization
}

\author{
Libo Liu ${ }^{1, *}$ and Weixing Wan $^{1}$ \\ (Manuscript received 11 January 2001, in final form 21 May 2001)
}

\begin{abstract}
A theoretical low-latitude ionospheric model was used to study the equatorial anomaly trough (EAT) and analyze factors which contribute to the variations of the EAT location. Model simulations showed significantly diurnal, seasonal and longitudinal variations in the EAT location. The EAT situated to the north of magnetic dip equator at June solstice and shifted to the south at December solstice. It was found that the seasonal variation of the EAT location was mainly caused by neutral winds, not by the seasonal variations in ionic photoionization production rates themselves. Distinct longitudinal variation in the EAT location occurred at the north of dip equator near $285^{\circ} \mathrm{E}$ and at the south of dip equator near $110^{\circ} \mathrm{E}$, which was qualitatively consistent with previous observations. Simulations showed that the longitudinal variation of the EAT location was caused mainly by the longitudinal structure in neutral winds. At most longitudes, the effects of magnetic declination and the displacement between geographical and magnetic equators were negligible. Only at regions near the $330^{\circ} \mathrm{E}$ longitudes did the magnetic declination play some larger influence on the EAT location, but it was still much smaller than that of neutral winds.
\end{abstract}

(Key Words: Low latitude ionosphere, Ionospheric model, Equatorial anomaly trough)

\section{INTRODUCTION}

A fascinating feature of low latitude ionosphere is that the latitude profiles of F2 peak electron density, or critical frequency, foF2, often show a minimum near the dip equator and two crests near magnetic dips $30^{\circ} \mathrm{N}$ and S, respectively. This is well known as the Equatorial Ionization Anomaly (EIA) or Appleton Anomaly. The mechanism of the EIA formation is the

\footnotetext{
${ }^{1}$ Wuhan lonospheric Observatory, Wuhan Institute of Physics and Mathematics, The Chinese Academy of Sciences, Wuhan, Hubei, P. R. China

* Corresponding author address: Dr. Libo Liu, Wuhan lonospheric Observatory, Wuhan Institute of Physics and Mathematics, The Chinese Academy of Sciences, Wuhan, Hubei, 430071, P. R. China; E-mail: lliu@wipm.whenc.ac.cn
} 
so-called "equatorial fountain effect", i.e., the plasma upward motion of the $\boldsymbol{E} \times \boldsymbol{B}$ vertical drift due to east electric field and geomagnetic field, and the field-aligned diffusions away from the dip equator caused by gravity, neutral wind and plasma pressure gradient. During the past 50 years, the EIA has been studied completely by experimental and theoretical methods. Unfortunately, the minimum centered near the dip equator in the latitude profiles of F2 peak electron density, i.e., the equatorial anomaly trough (EAT), escaped the scientists' attention.

Some papers mention the evolution of the EAT. Lyon and Thomas (1963) found that the latitude of the EAT moved with local time, and Huang (1986) studied the EAT based on the ISS-b and Alouette satellite data. He pointed out that there were clearly seasonal and longitudinal variations in the EAT location. This paper focused on the behavior of the EAT location. A low-latitude ionospheric model (Liu et al. 2000) was used to investigate effects of some physical factors (ionic photoionization production rates, neutral winds, the differences between the geographic and magnetic coordinates) on the variation in EAT location.

\section{A BRIEF DESCRIPTION OF THE MODEL AND ITS INPUTS}

The two-dimensional low latitude ionospheric model used in this paper was an update one of Tu et al. (1997), as previously described by Liu et al. (2000). In the model, the concentrations and velocities of ions $\left(\mathrm{O}^{+}, \mathrm{H}^{+}, \mathrm{He}^{+}, \mathrm{N}_{2}^{+}, \mathrm{O}_{2}{ }^{+}\right.$and $\left.\mathrm{NO}^{+}\right)$were calculated from the time dependent continuity and momentum equations within a geomagnetic meridian plane. The energy equations were not solved in the model, and the electron and ion temperatures were calculated from IRI90 (Biltza 1990). Under the assumption of charge neutrality, in the ionosphere the electronic concentration was the sum of all ion species. The plasma momentum equations included the dynamic couplings through collision interactions between ion and neutrals or ions, $\boldsymbol{E} \times \boldsymbol{B}$ drifts, and field-aligned diffusions due to plasma pressure gradient and gravity. In the model, the transport equations were processed by employing an operator splitting scheme and were solved within a magnetic meridian plane. The discrete grid points, which did not move during the calculations, were taken as the cross points of geomagnetic field lines and plasma vertical drift lines. The boundary conditions at the base altitude, 130 $\mathrm{km}$, were chemical equilibrium conditions and at the uppermost points were linear extrainterpolations. And, the influence of boundary conditions on the $\mathrm{F}$ region was negligible. The model was calculated for 72 hours with the same day's parameters to diminish the influence of initial conditions, and the final 24-hour data were outputted for diagnostics.

Solar EUV photoionizations of $\mathrm{O}^{+}, \mathrm{He}^{+}, \mathrm{N}^{+}$and $\mathrm{O}_{2}^{+}$and chemical reactions related to those ionized species (including the 4S, 2D and 2P state divisions of $\mathrm{O}^{+}$) were taken into account. The EUV fluxes were from the EUVAC model (Richards et al. 1994). The concentrations and temperatures of the neutral species in the ionospheric model were provided by MSIS86 (Hedin 1986), except for the concentration of the constituent NO which was calculated using the expression of Mitra (1968), the plasma $\boldsymbol{E} \times \boldsymbol{B}$ vertical drift velocities were from the ISR drift model (Richmond et al. 1980), and the horizontal neutral winds were taken from HWM90 (Hedin et al. 1990). The HWM winds are not necessarily accurate, but they are the best we have at the moment. According to Anderson (1981), the influence of the zonal $\boldsymbol{E} \times \boldsymbol{B}$ drift on the densities of F-layer ionosphere can be ignored. Therefore, the east-west $\boldsymbol{E} \times \boldsymbol{B}$ drift was 
set as zero in the calculations. Results from the model generally agreed well with measurements (Tu et al. 1997).

Model calculations in the next section were carried out using our ionospheric model under quiet magnetic activity and solar-medium conditions (Solar $10.7 \mathrm{~cm}$ flux index, $\mathrm{F}_{107}=99$, its 81 days average index, $F_{107 \mathrm{~A}}=101$, and $\mathrm{Ap}=6$ ).

\section{RESULTS AND DISCUSSION}

Many studies show that the EIA in foF2 was usually formed at around 1000-1200LT and disappear at near 2100-2300LT for middle and low solar activity, while it would appear earlier and disappeared later for high solar activity. The data in the interval of 1200-2200LT was taken for analysis in this paper.

\subsection{The Seasonal Variation}

To investigate the seasonal variation of EAT location, we took the longitude of $110^{\circ} \mathrm{E}$ as an example and changed day number, iyd, from 1 to 365 in the model calculations. Figure 1 depicts the magnetic latitude of EAT location at longitude $110^{\circ} \mathrm{E}$ as a function of day number and local time. The EAT was located at the north side of dip equator at June solstice, and it reached $3^{\circ}-5^{\circ} \mathrm{N}$. At December solstice, it shifted to the south side of dip equator. This was consistent with previous observations (Huang 1986). At the development stage, the EAT generally moved away from dip equator. At about $1400 \mathrm{LT}$ the tendency reversed, and the EAT moved toward dip equator. But, it shifted away from dip equator again after a short time. But, during the equinox daytime, the EAT location varied little with local time.

To find out the factor(s) mainly responsible for the seasonal variation of EAT location, we isolated the seasonal variations of neural winds, densities of neutral species and ionic photoionization productions due to the solar declination change. The effects of these factors themselves on the EAT location are depicted in Fig. 2.

The seasonal variations of ionic photoionization productions were induced by the changes in the solar declination and number densities of neutral atmosphere. Figure 2A shows that the seasonal variations of ionic photoionization productions controlled by the solar declination influenced the EAT location very little. The solar declination varied from $-23.45^{\circ}$ to $23.45^{\circ}$ in a year. Under the direct influence of the solar declination change, the EAT location shifted less than $0.2^{\circ}$ at most local times, and the maximum was only 0.6. Although the observations showed that the EAT was located the most north during the June (Fig. 1), the effects of ionic photoionization production change due to solar declination on the seasonal variation in EAT location was ignored (Fig. 2A).

Taking the solar declination for the value of day 83 , the isolated contribution of seasonal variation of neutral winds on the EAT location is plotted in Fig. 2B. As Fig. 2B shows, the principal characteristics of the seasonal variation of EAT location was formed by the seasonal variation in neutral horizontal winds. Further calculations showed that the contribution of the densities of neutral species was much smaller than that of neutral winds (Figure not presented here). 


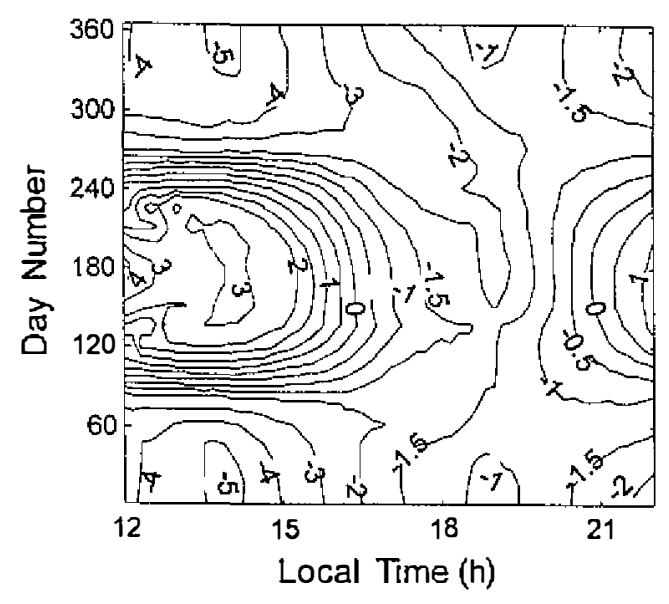

(a)

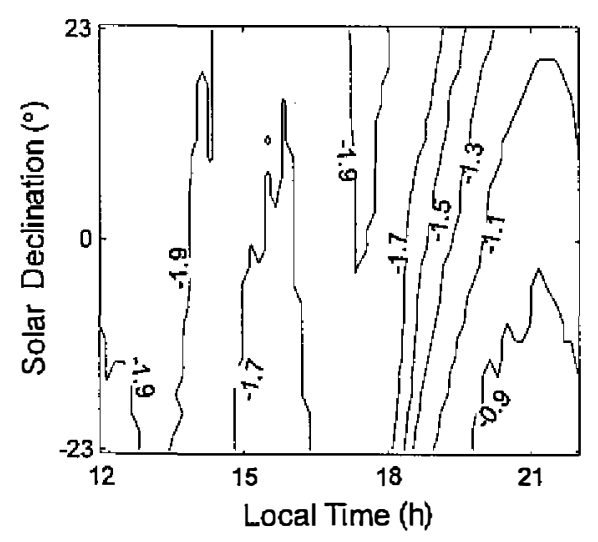

Fig. 2a. The effects of seasonal variations of the ionic photoionization production due to solar declination change on the location of calculated equatorial trough.

Fig. 3. The variation of calculated equatorial trough position as functions of geographic longitude and local time on a given day (the 83rd day).
Fig. 1. The variation of calculated equatorial trough location (magnetic latitude) at longitude $110^{\circ} \mathrm{E}$ as functions of day number and local time.

(b)

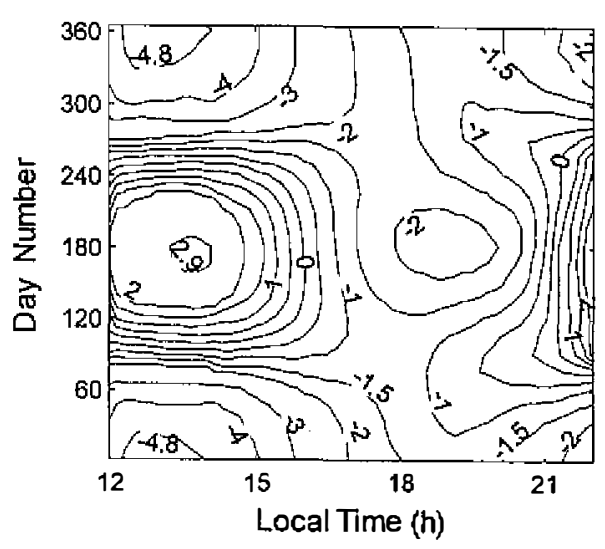

Fig. $2 b$. The effects of neutral winds on the location of calculated equatorial rough.

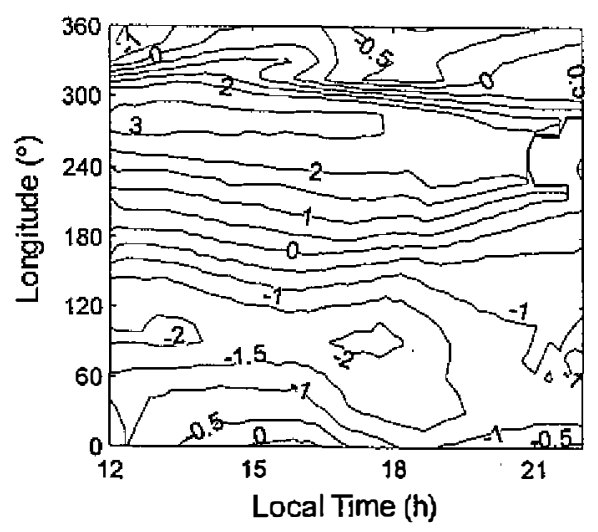




\subsection{The Longitudinal Variation}

Consider the 83rd day. The calculated EAT locations as a function of longitude are plotted in Fig. 3. It shows that there was distinct longitudinal variation in the EAT location. The EAT was at north, farthest from magnetic equator at near $285^{\circ} \mathrm{E}$ and south, nearest $110^{\circ} \mathrm{E}$ respectively.

(a)

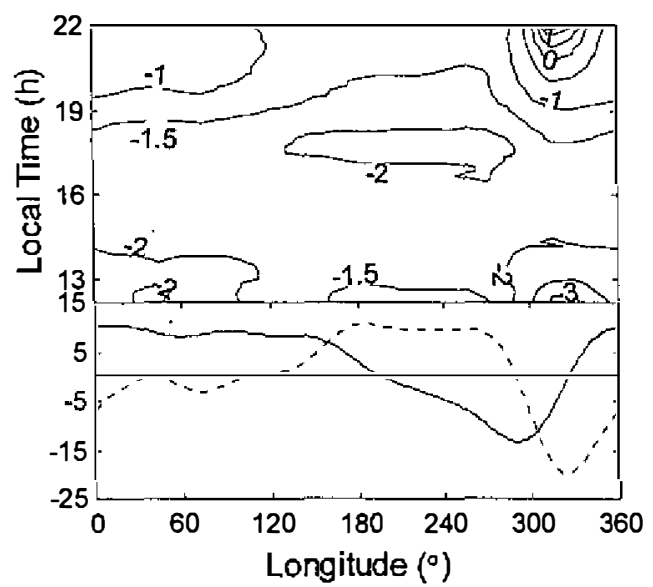

(b)

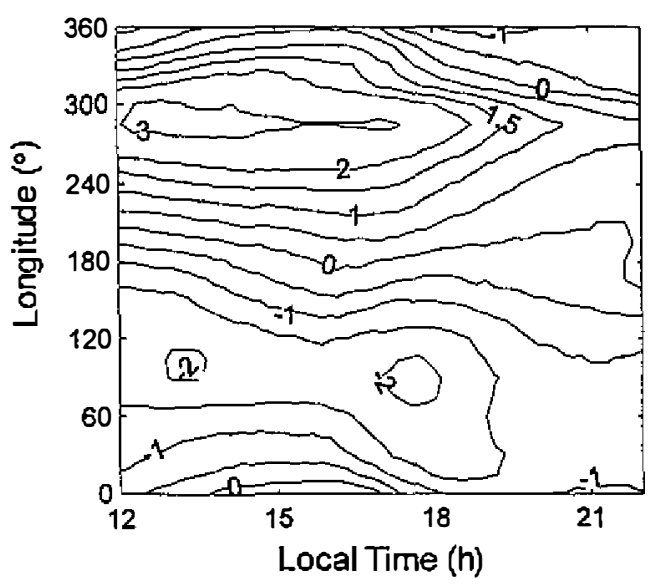

Fig. 4. (a) The variation of calculated equatorial trough position due to the longitudinal variations of declination angle and the displacement between the magnetic dip equator and geographic equator. Plotted in the bottom part of the left panel are the longitudinal variations of geographic latitudes (solid line) and magnetic declinations (dotted line) at the magnetic dip equator according to IGRF95 geomagnetic empirical model. (b) The effects of the longitude structure in neutral winds on the EAT location.

The factors controlling the longitudinal variation of EAT possibly included the longitudinal structure in neutral atmosphere (winds and number densities of neutral species), magnetic declination and the displacement between the geographic and magnetic coordinates. The effects of these factors on EAT location are shown in Fig. 4A and B respectively. Figure 4A shows the influences on the EAT location by the longitudinal structure of magnetic declination and the displacement between geographic and magnetic coordinates. The geographic latitude of dip equator and its magnetic declination are also plotted in the bottom panel of Fig. 4A. In Fig. 4A, results indicate clearly that the displacement between the geographic and magnetic coordinates itself influenced the longitudinal structure of the location of EAT very limited. That is also the case for the magnetic declination, except near the region of $320^{\circ} \mathrm{E}$. In the region of $320^{\circ} \mathrm{E}$, the shift amplitude of EAT location was larger somewhat, and it was about $1^{\circ}$ during daytime and $2.5^{\circ}$ maximum at nighttime.

Then, the values of magnetic declinations and dip angles were taken as those of longitude $110^{\circ} \mathrm{E}$, the variation of the location of EAT caused by the longitudinal structure of neutral 
winds is plotted in Fig. 4B. Compared with Fig. 3 and Fig. 4B, it was found that the longitudinal structure of neural winds mainly contributed to the longitudinal feature in the EAT location, not by longitudinal variation in the magnetic declination and the displacement between geographic and magnetic coordinates.

\section{SUMMARY}

During the quiet magnetic activity period, the main factors influencing the EAT location included the background neutral atmospheric parameters (number densities, temperature and horizontal winds), magnetic declination, the displacement between magnetic and geographic coordinates, and ionic production rates due to the change in solar declination, etc.

The model calculations showed that the EAT location varied with the local time, season and longitude. During the June solstice, the EAT generally was located at the north side of dip equator, and it shifted to the south side of dip equator in December solstice. The EAT was located at the most south near longitude $100^{\circ} \mathrm{E}$, and was located the most north near $285^{\circ} \mathrm{E}$. These were basically consistent with the observations of Huang (1986).

We adjusted each of them respectively to investigate the effects of these factors on the seasonal and longitudinal variations of EAT location. The results showed that among the above factors, the seasonal variation of horizontal neutral winds played the most important role in the seasonal variation of the EAT location. The effect of the variation in ionic photoionization productions due to the change of solar declination was negligible. The influence of the densities and temperature of neutral atmosphere was much less distinct than that of horizontal neutral winds.

Results show that the background horizontal neutral winds played the most significant role in the longitudinal variation of the EAT location. The displacement between dip equator and geographic equator itself had a negligible influence on the EAT position. In most longitude sectors, the effect of the magnetic declination itself also can be ignored except near $330^{\circ} \mathrm{E}$. And, the magnetic declination can not reproduce the basic picture of longitudinal structure in the EAT location. The simulations did not support that the apparent longitude variation of EAT location was largely attributable to the magnetic declination effect of Huang (1986).

It is well known that the trough and crests of the equatorial anomaly are caused by plasma fountain. The $\boldsymbol{E} \times \boldsymbol{B}$ drift drives ionization from the magnetic equator across the magnetic field lines to higher altitudes, in the absence of neutral winds, causing almost identical effects in the two hemispheres (Balan and Bailey 1995). Some studies (e.g., Balan and Bailey 1995; Balan et al. 1995) have revealed that neutral winds, causing asymmetries in the otherwise symmetrical equatorial fountain, are mainly responsible for the conjugate-hemisphere differences at low latitudes. One can infer that the asymmetrical effects of neutral winds form the differences of the north-south crests of the EIA and the displacement of EAT and the dip equators. In other words, the motion of the crests and the trough are correlated to some extent. The detailed correlation between the EIA and EAT will be investigated using the foF2 atlas of Japanese topside ionospheric sounding satellite ISS-b. It should be pointed out that because the magnetic field in our ionospheric model is under the dipole field assumption, the displacement between the dip equator and the equatorial electrojet can not be taken into account. So, 
its effects on the EAT location were not considered in this paper.

Acknowledgement This research is supported by the Natural Science Foundation of China (49804008, 49974039).

\section{REFERENCES}

Anderson, D. N., 1981: Modeling the ambient, low latitude F-region ionosphere-a review. $J$. Atmos. Terr. Phys., 43, 753-762.

Balan, N., G. J. Bailey, R. J. Moffett, Y. Z. Su, and J. E. Titheridge, 1995: Modelling studies of the conjugate-hemisphere differences in ionospheric ionization at equatorial anomaly latitudes. J. Atmos. Terr. Phys., 57, 279-292.

Balan, N., and G. J. Bailey, 1995: Equatorial plasma foutain and its effects: possibiltity of an additional layer. J. Geophys. Res., 100, 21421-21432.

Bilitza, D., 1990: International reference ionosphere 1990, NSSDC/WDC-A-R\&S, 90-92.

Fejer, B. G., 1981: The equatorial ionospheric electric fields-A review. J. Atmos. Terr. Phys., 43, 377-386.

Hedin, A. E., 1987: MSIS-86 thermospheric model. J. Geophys. Res., 92, 4649-4662.

Hedin, A. E., M. A. Biondi, R. G. Burnside, G. Hernandez, R. M. Johnson, T. L. Killeen, C. Mazaudier, J. W. Meiwether, J. E. Salah, R. J. Sica, R. W. Smith, N. W. Spencer, V. B. Wickwar, and T. S. Virdi, 1991: Revised global model of thermosphere winds using satellite and ground-based observations. J. Geophys. Res., 96, 7657-7688.

Huang, C-M., 1986: On the equatorial trough of F2-layer ionization. J. Atmos. Terr. Phys., 48 (6), 579-583

Liu, L., W. Wan, J. Tu, and Z. Bao, 2000: Modeling of the ionospheric response to the solar eclipse of 24 october 1995. TAO, 11, 543-554.

Lyon, A. J, and L. Thomas, 1963: The F2-region equatorial anomaly in the African, American and East Asian sectors during sunspot maximum. J. Atmos. Terr. Phys., 25, 373-386.

Mitra, A. P., 1968: A review of D-region process in non-polar latitudes. J. Atmos. Terr. Phys., 30, 1065-1114.

Richmond, A. D., M. Blanc, B. A. Emery, R. H. Wand, B. G. Fejer, R. F. Woodman, S. Ganguly, P. Amayeru, R. A. Behnke, C. Calderon, and J. V. Evans, 1980: An empirical model of quiet-day ionospheric electric fields at middle and low latitudes. J. Geophys. Res., 85(A9), 4658-4664

Richards, P. G., J. A. Fennelly, and D. G. Torr, 1994: EUVAC: A solar EUV flux model for aeronomic calculations. J. Geophys. Res., 99(A5), 8981-8992.

Tu, J., L. Liu, and Z. Bao, 1997: A low latitude ionospheric theoretical model. Chinese J. Space Phys., 17, 212-219 (in Chinese). 